\title{
Comparison of Elite Athletes and Essential Hypertension Patients for Angiotensin-Converting Enzyme (ACE) I/D and $A C E$ G2350A Gene Polymorphisms
}

\author{
Tuba Gunel ${ }^{* 1}$, Candan Eker ${ }^{1 *}$, Ece Gumusoglu ${ }^{1}$, Sebnem Ozdemir ${ }^{2}$, Eser Durmaz ${ }^{3}$, Damla Koca ${ }^{3}$, \\ Kilic Aydinli ${ }^{4}$ \\ ${ }^{1}$ Istanbul University, Faculty of Science, Department of Molecular Biology and Genetics, Turkey \\ ${ }^{2}$ Istinye University, Faculty of Economics, Administrative and Social Sciences, Department of Management Information Systems, \\ Turkey \\ ${ }^{3}$ Istanbul University-Cerrahpasa, Cerrahpasa Medical Faculty, Department of Cardiology, Turkey \\ ${ }^{4}$ Medicus Health Center, 34365 Istanbul, Turkey \\ *Both authors are equally contributed
}

*Corresponding author: Tuba Gunel, Istanbul University, Faculty of Science, Department of Molecular Biology and Genetics, Turkey

ARTICLE INFO

Received: 蔧 September 25, 2020

Published: 慧 October 06, 2020

Citation: Tuba Gunel, Candan Eker, Ece Gumusoglu, Sebnem Ozdemir, Eser Durmaz, Damla Koca, Kilic Aydinli. Comparison of Elite Athletes, Essential Hypertension Patients for AngiotensinConverting Enzyme (ACE) I/D and ACE G2350A Gene Polymorphisms. Biomed J Sci \& Tech Res 31(1)-2020. BJSTR. MS.ID.005032.

Keywords: $\quad$ rs4343 (ACE G2350A); rs1799752 (ACE I/D) Polymorphisms; Elite Athlete; Sports Performance; Hypertension

\section{ABSTRACT}

Objective: Angiotensin-converting enzyme (ACE) gene produces an ACE protein which is the key component of the renin-angiotensin system and contains several polymorphisms which affects the physical performance that is significantly important for athletes. Insertion/deletion (I/D) and G2350A polymorphisms which have one of the most powerful influences in the $A C E$ gene is known to regulate cardiovascular mechanisms by taking a role in angiotensin-system.

Methods: The present study aimed to investigate the genetic association and haplotypes formed by rs1799752 (ACE I/D) and rs4343 (ACE G2350A) polymorphisms in essential hypertension patients, elite athletes and healthy controls in total 79 subjects (27 hypertension patients, 27 elite athletes and 25 control group individuals) from Turkish population. In this study, $A C E$ I/D and G2350A polymorphisms were genotyped by using polymerase chain reaction and restriction fragment length polymorphism (PCR-RFLP) method and identified by bioanalyzer after restriction digestion.

Results: The prevalence of genotype frequencies of the ACE rs1799752 II/ID/DD was $3.7 \%, 44.44 \%, 51.85 \%$ in hypertension patients; $3.7 \%, 33.33 \%, 62.96 \%$ in elite athletes and $12 \%, 24 \%, 64 \%$ in healthy controls, respectively. Also, the prevalence of genotype frequencies of the $A C E$ rs $4343 \mathrm{GG} / \mathrm{GA} / \mathrm{AA}$ was $7.41 \%, 77.78 \%, 14.81 \%$ in hypertension patients; $0 \%, 77.78 \%, 22.22 \%$ in elite athletes and $4 \%, 84 \%, 12 \%$ in healthy controls, respectively.

Conclusion: Our data suggest that "D" allele for I/D and "A" allele for G2350A polymorphisms of $A C E$ gene may have a potential role with a large number of patients and control groups in Turkish population.

\section{Introduction}

The people who make regular and professional sports have different structured and worked of hearts which is called the athlete's heart. This difference in the cardiac structure and function of the heart causes cardiovascular diseases such as hypertension.
Thickening of the ventricular wall due to an increase in ventricular volume and hypertrophy of myocardium is an example of cardiac structural changes, besides it is known to show an increase in stroke volume and decrease in resting heart rate as to cardiac 
function $[1,2]$. The hearts of those who use muscle strength such as runners, aerobic exercisers and wrestlers are changing and evolving. These changes occur not only by exercise but also by the influence of genetic factors. There are a variety of genes related to sport performance and athlete's heart structure. Moreover, they are likely to be polygenic because there are hundreds of genetic variance combinations among individuals. Genetic diversity is the reason why individuals are different in developing species. In genes, polymorphisms appear as minor genetic variations that have led to genetic diversity in the genetic code. These changes should be seen in more than $1 \%$ of the population to be called polymorphism [3]. The polymorphisms affect the sport performance via changing the significant gene functions especially the angiotensin-converting enzyme gene.

The ACE gene is one of the most studied candidate genes related to hypertension. $A C E$ is a zinc-dependent dipeptidyl carboxypeptidase that plays an important role in the regulation of blood pressure by converting "angiotensin I" peptide into a potent vasoconstrictor peptide "angiotensin II", while it also inactivates the vasodepressor bradykinin to eventually promote the growth of cardiac cells, the biggest number of studies have been performed to change in cardiac structure and function such as left ventricular hypertrophy caused by a complication of cardiovascular diseases such as essential hypertension as well as left ventricular hypertrophy shown for athlete groups [2,4]. ACE gene that encodes the angiotensin-converting enzyme is located in the $\mathrm{q}$ arm of the chromosome 17, and it has 26 exons and 25 introns. According to NCBI records, $A C E$ gene has more than 160 polymorphisms which are mainly single nucleotide polymorphisms (SNP) [5]. Among the polymorphisms of the ACE gene, the G2350A polymorphism (rs4343) is known to have the most significant effects on the plasma angiotensin-converting enzyme (ACE) concentration, although the most widely studied polymorphism of $A C E$ gene is insertiondeletion of $287 \mathrm{bp}$ existing in intron 16 of this gene [2]. It has been demonstrated for an athlete group that G2350A polymorphism of $A C E$ gene can cause alterations in cardiac structure and function [6]. Although there are several studies have been conducted to evaluate the correlation between ACE G2350A polymorphism and hypertension, it was found conflicting results in those previous studies [7-9]. Therefore, we aimed to perform an association study to evaluate the allelic and genotypic frequencies of both (ACE I/D, $A C E$ G2350A) polymorphisms and their prevalence on essential hypertension and sports performance.

\section{Materials and Methods}

\section{Ethical Approval}

The study was approved by the Ethics Committee of Istanbul University (Istanbul, Turkey; Project no. 2011/2115-897). The study protocol was performed according to the Declaration of Helsinki and the institutional review board-approved protocols for human study participants at the Istanbul University. All the participants provided written informed consent.

\section{Study Design}

The study group was designed according to the three different experimental groups for a total of 79 subjects consisting of 27 essential hypertensive patients ( $H$, group 1), 27 elite athletes (E, group 2) and 25 unrelated healthy individuals as controls (C, group $3)$. The mean age range of hypertension patients, elite athletes and controls were $39 \pm 6.2,15 \pm 3.1$ and $28 \pm 5.5$, respectively. All hypertension subjects are followed up and evaluated at the outpatient clinics in Istanbul University-Cerrahpasa, Cardiology Department of Cerrahpasa Medical Faculty (Istanbul, Turkey). Unrelated healthy controls and elite athletes were collected randomly to this research study and had no history of hypertension. Also, control individuals and hypertension patients were not engaged in sports as a professional level. All study participants are ethnically Caucasian and geographically different residents of Turkey.

\section{Genotyping of rs1799752 (ACE I/D) and rs4343 (ACE G2350A) Polymorphisms}

$2 \mathrm{ml}$ of peripheral blood samples were collected in EDTAcontaining tubes from Istanbul University-Cerrahpasa, Cerrahpasa Faculty of Medicine, Department of Cardiology and transferred to Istanbul University, Faculty of Sciences, Department of Molecular Biology and Genetics, Genetical Diseases and Molecular Analysis Laboratory (Istanbul, Turkey). Total genomic DNA was isolated and purified from peripheral blood lymphocytes following the manufacturer's instructions by using DNA Extraction kit (Agilent Technologies Inc. Santa Clara, CA, USA). The quantity and quality of DNA samples were measured by NanoPhotometer, P330 (Implen GmbH, Munich, Germany). Purified DNA samples were used for genotyping of the rs1799752 (I/D) and rs4343 (G2350A) polymorphisms in the ACE gene.

ACE (I/D) and ACE (G2350A) polymorphisms were genotyped by PCR-RFLP methods by using the primer pairs (ACE (I/D) primers: 5'-CTGGAGACCACTCCCATCCTTTCT-3' (forward primer) and 5'- GATGTGGCCATCACATTCGTCAGAT-3' (reverse primer), ACE (G2350A) primers: 5'-CTGACGAATGTGATGGCCGC-3' (forward primer) and 5'-TTGATGAGTTCCACGTATTTCG-3' (reverse primer)). $A C E$ I/D and G2350A polymorphism regions were amplified using $200 \mathrm{ng} / \mu \mathrm{l}$ genomic DNA as template. PCR was performed in a total volume of $50 \mu \mathrm{l}$ reaction mixture containing $20 \mathrm{pmol}$ of two primer pairs, $0.02 \mu \mathrm{mol}$ each dNTP, $1.5 \mathrm{mmol} \mathrm{l}-1 \mathrm{MgCl} 2,5 \mu \mathrm{l} 10 \times$ Taq buffer and 1.5 unit of Taq DNA polymerase (AmpliKIT, Novazym, Poland). PCR was performed according to the following conditions: initial denaturation step at $95{ }^{\circ} \mathrm{C}$ for $5 \mathrm{~min}, 35$ cycles of denaturation at $94{ }^{\circ} \mathrm{C}$ for $30 \mathrm{sec}$, annealing at $58{ }^{\circ} \mathrm{C}$ for $30 \mathrm{sec}$, extension at $72{ }^{\circ} \mathrm{C}$ for $30 \mathrm{sec}$ and a final step of $10 \mathrm{~min}$ at $72{ }^{\circ} \mathrm{C}$ was carried out on 
Agilent Sure Cycler 8800 instrument (Agilent Technologies. Inc. Santa Clara, CA, USA). The amplified PCR products were digested with $10 \mathrm{U} / \mu \mathrm{l}$ of DdeI (ACE I/D) and BstUI (ACE G2350A) restriction enzymes (Thermo Fisher Scientific, MA, USA) at $65^{\circ} \mathrm{C}$ for $3 \mathrm{~h}$.

The restricted products were analyzed qualitatively and quantitatively by Agilent 1000 Bioanalyzer instrument (Agilent 1000; Agilent Technologies, Inc.). Expected fragment lengths were checked on bioanalyzer results (ACE (II) genotype: $490 \mathrm{bp}$ fragment, $A C E$ (DD) genotype: $180 \mathrm{bp}$ fragment, $A C E$ (ID) genotype: $180 \mathrm{bp}$ and 490bp fragments, ACE (G2350A) genotype: 100bp and 22bp fragments according to the reference ACE (GG) genotype of $122 \mathrm{bp}$ fragment). For quality control, the genotyping was repeated once with a concordance rate of $100 \%$ in the samples included in the study. All experiments were performed in the Genetical Diseases and Molecular Analysis Unit of Istanbul University (Istanbul, Turkey).

\section{Statistical Analysis}

Statistical analysis of the study was conducted with R Console 3.4.1., RGui platform (i386, 64-bit). The frequency of genotypes and alleles was determined by direct gene counting method. Odds ratio (OR) and 95\% confidence intervals (CIs) were calculated for the association between ACE (I/D) and ACE (G2350A) polymorphisms in hypertension patients, elite athletes and controls, respectively. Pearson's chi-square $\left(\chi^{2}\right)$ test was performed to compare the genotype distribution and allele frequency between groups. Chisquare independence test was used to investigate the significance of the distribution of hypertension patients, elite athletes and healthy controls. All P-values were two-sided and P-value $<0.05$ was considered to represent a statistically significant result.

\section{Results}

\section{Study Group}

The study group consisted of a total of 79 subjects with $\mathrm{H}(\mathrm{n}=$ $27), E(n=27)$ and $C(n=25)$. Samples with age $15-39$ years were obtained in this study and there was no statistically significant differences between age, gender and age of onset distributions ( $p>$ $0.05)$. The average blood pressure parameters of the subjects and controls are summarized in (Table 1).

Table 1: Average blood pressure parameters of the $\mathrm{H}, \mathrm{E}$ and $\mathrm{C}$ subjects.

\begin{tabular}{|c|c|c|c|}
\hline $\begin{array}{c}\text { Mean age and BP } \\
\text { parameters }\end{array}$ & $\begin{array}{c}\text { H (Group 1) } \\
n=27\end{array}$ & $\begin{array}{c}\text { E (Group 2) } \\
n=27\end{array}$ & $\begin{array}{c}\text { C (Group 3) } \\
n=25\end{array}$ \\
\hline Age (year) & $39 \pm 6.2$ & $15 \pm 3.1$ & $28 \pm 5.5$ \\
\hline $\begin{array}{c}\text { Systolic blood } \\
\text { pressure (mm Hg) }\end{array}$ & $\begin{array}{c}139.19 \pm \\
29.58\end{array}$ & $118.25 \pm 12.77$ & $117.32 \pm 10$ \\
\hline $\begin{array}{c}\text { Diastolic blood } \\
\text { pressure (mm Hg) }\end{array}$ & $\begin{array}{c}87.43 \pm \\
16.20\end{array}$ & $72.32 \pm 09.80$ & $68.24 \pm 08.90$ \\
\hline
\end{tabular}

\section{Genotype and allele frequencies of ACE I/D and ACE G2350A Polymorphisms in $\mathrm{H}, \mathrm{E}$ and C Groups}

The genotype and allele frequencies of the ACE I/D (rs1799752) polymorphism in the study groups are presented in (Table 2).

Table 2: Genotype and allele frequencies of rs1799752 (ACE I/D) polymorphism in hypertension patients, elite athletes and healthy controls.

\begin{tabular}{|c|c|c|c|c|c|c|c|c|c|}
\hline \multirow[b]{2}{*}{$\begin{array}{c}\text { ACE } \\
\text { polymorphism }\end{array}$} & \multirow[b]{2}{*}{$\begin{array}{c}\text { Hypertension } \\
\text { Patients (H) } \\
\text { N (Frequency \%) } \\
\text { (n= 27) }\end{array}$} & \multirow{2}{*}{$\begin{array}{c}\begin{array}{c}\text { Elite athletes } \\
\text { (E) }\end{array} \\
\text { N (Frequency } \\
\%) \\
(n=27)\end{array}$} & \multirow[b]{2}{*}{$\begin{array}{c}\text { Controls (C) } \\
\text { N (Frequency \%) } \\
(n=25)\end{array}$} & \multicolumn{3}{|c|}{ Hypertension Patients vs. Controls } & \multicolumn{3}{|c|}{ Elite athletes vs. Controls } \\
\hline & & & & OR $(95 \% \mathrm{CI})$ & $\chi^{2}$ & $\mathbf{P}$ & OR $(95 \% \mathrm{CI})$ & $\chi^{2}$ & $\mathbf{P}$ \\
\hline \multicolumn{10}{|c|}{ rs1799752, Insertion (I) / Deletion (D) } \\
\hline \multicolumn{10}{|c|}{ Genotype } \\
\hline II & $1(3.70 \%)$ & $1(3.70 \%)$ & $3(12 \%)$ & \multicolumn{3}{|c|}{ Reference } & \multicolumn{3}{|c|}{ Reference } \\
\hline ID & $12(44.44 \%)$ & $9(33.33 \%)$ & $6(24 \%)$ & $\begin{array}{c}6 \\
(1.040-16.783)\end{array}$ & 2.027 & 0.154 & $\begin{array}{c}4.5 \\
(1.04-17.201)\end{array}$ & 1.404 & 0.236 \\
\hline DD & $14(51.85 \%)$ & $17(62.96 \%)$ & $16(64 \%)$ & $\begin{array}{c}2.625 \\
(1.038-15.104)\end{array}$ & 0.634 & 0.425 & $\begin{array}{l}3.187 \\
(1.038- \\
14.929)\end{array}$ & 0.923 & 0.336 \\
\hline \multicolumn{10}{|c|}{ Allele } \\
\hline I & $14(25.93 \%)$ & $11(20.37 \%)$ & $12(24 \%)$ & \multicolumn{3}{|c|}{ Reference } & \multicolumn{3}{|c|}{ Reference } \\
\hline $\mathrm{D}$ & $40(74.07 \%)$ & $43(79.63 \%)$ & $38(76 \%)$ & $\begin{array}{c}0.902 \\
(1.014-2.766)\end{array}$ & 0.051 & 0.821 & $\begin{array}{c}1.234 \\
(1.014-2.887)\end{array}$ & 0.197 & 0.657 \\
\hline
\end{tabular}


For rs1799752 (ACE I/D) polymorphism; using genotype ACE (II) as a reference, although the frequency of genotype ACE (II) was equal between hypertension patients and elite athletes according to the controls $(3.70 \%(\mathrm{H}), 3.70 \%(\mathrm{E}), 12 \%(\mathrm{C})$, respectively), the frequency of $A C E$ (ID) genotype in hypertension patients and elite athletes was higher than healthy controls $(44.44 \%$ (H) vs. $24 \%$ (C), 33.33\% (E) vs. $24 \%$ (C), Table 2). Also, "D" allele was more prevalent when compared to the "I" allele in all groups. However, the frequency of rs1799752 ACE "D" allele had no significant differences between $\mathrm{H}$ vs. C $\left(\chi^{2} 1=0.051, \mathrm{p}=0.821, \mathrm{OR}=0.902\right.$, $95 \% \mathrm{CI}=1.014-2.766)$ and $\mathrm{E}$ vs. $\mathrm{C}\left(\chi^{2} 1=0.197, \mathrm{p}=0.657, \mathrm{OR}=\right.$ $1.234,95 \% \mathrm{CI}=1.014-2.887)$.

(Table 3) indicates the genotype and allelic frequencies of the ACE G2350A (rs4343) polymorphism in the study group.

Table 3: Genotype and allele frequencies of rs4343 (ACE G2350A) polymorphism in hypertension patients, elite athletes and healthy controls.

\begin{tabular}{|c|c|c|c|c|c|c|c|c|c|}
\hline \multirow[b]{2}{*}{$\begin{array}{c}\text { ACE } \\
\text { polymorphism }\end{array}$} & \multirow{2}{*}{$\begin{array}{c}\text { Hypertension } \\
\text { Patients (H) N } \\
\text { (Frequency \%) } \\
(n=27)\end{array}$} & \multirow{2}{*}{$\begin{array}{c}\text { Elite } \\
\text { athletes } \\
\text { (E) N } \\
\text { (Frequency } \\
\text { \%) (n=27) }\end{array}$} & \multirow{2}{*}{$\begin{array}{c}\text { Controls (C) } \\
\text { N } \\
\text { (Frequency } \\
\% \text { ) }(\mathrm{n}=25)\end{array}$} & \multicolumn{3}{|c|}{ Hypertension Patients vs. Controls } & \multicolumn{3}{|c|}{ Elite athletes vs. Controls } \\
\hline & & & & OR $(95 \% \mathrm{CI})$ & $\chi^{2}$ & $\mathbf{P}$ & $\begin{array}{l}\text { OR ( } 95 \% \\
\text { CI) }\end{array}$ & $\chi^{2}$ & $\mathbf{P}$ \\
\hline \multicolumn{10}{|c|}{ rs4343, G2350A } \\
\hline \multicolumn{10}{|c|}{ Genotype } \\
\hline GG & $2(7.41 \%)$ & $0(0.00 \%)$ & $1(4 \%)$ & Reference & & & Reference & & \\
\hline GA & $21(77.78 \%)$ & $21(77.78 \%)$ & $21(84 \%)$ & $\begin{array}{c}0.5 \\
(1.04-16.961)\end{array}$ & 0.301 & 0.583 & *NA & - & - \\
\hline $\mathrm{AA}$ & $4(14.81 \%)$ & $6(22.22 \%)$ & $3(12 \%)$ & $\begin{array}{c}0.666 \\
(1.046-25.411)\end{array}$ & 0.079 & 0.778 & *NA & - & - \\
\hline \multicolumn{10}{|c|}{ Allele } \\
\hline G & $25(46.3 \%)$ & $21(38.89 \%)$ & $23(46 \%)$ & Reference & & & Reference & & \\
\hline A & $29(53.70 \%)$ & $33(61.11 \%)$ & $27(54 \%)$ & $\begin{array}{c}0.988 \\
(1.012-2.416)\end{array}$ & 0.00094 & 0.975 & $\begin{array}{c}1.338 \\
(1.012-2.44)\end{array}$ & 0.535 & 0.464 \\
\hline
\end{tabular}

Note: *Because of there has no GG genotype for Elite athletes, odds ratio and statistically related calculations could not have done for comparison of elite athletes vs. controls.

For rs4343 (ACE G2350A) polymorphism; using genotype ACE (GG) as a reference, the frequency of genotype $A C E(\mathrm{GA})$ between hypertension patients and elite athletes was equal and there were similar genotype frequencies between all groups $(77.78 \%$ vs. $84 \%$, $\chi^{2} 1=0.301, \mathrm{p}=0.583, \mathrm{OR}=0.5,95 \% \mathrm{CI}=1.04-16.961, \mathrm{H}$ vs. $\left.\mathrm{C}\right)$. Because of there were not ACE (GG) genotype carriers for elite athletes, odds ratio and statistically related calculations could not have done for the genotype frequency distribution of E vs. C $(77.78 \%$ vs. $84 \%$, OR (95\%CI): NA, Table 3). However, the frequency of $A C E$ (AA) genotype of elite athletes was more prevalent than in patients with hypertension and controls $(22.22 \%$ (E) vs. $12 \%$ (C); $22.22 \%$
(E) vs.14.81\% (H)). The frequency of rs4343 allele "A" was similar in between $\mathrm{H}$ vs. C $\left(53.70 \%\right.$ vs. $54 \%, \chi^{2} 1=0.00094, \mathrm{p}=0.975$, OR $=0.988,95 \% \mathrm{CI}=1.012-2.416)$. On the other hand, although the frequency of rs 4343 allele A was less frequent in healthy controls than in elite athletes (54\% (C) vs. $61.11 \%$ (E)), there was not found any significant differences between this group $\left(\chi^{2} 1=0.535, \mathrm{p}=\right.$ $0.464, \mathrm{OR}=1.338,95 \% \mathrm{CI}=1.012-2.44$, E vs. C, Table 3). However, $A C E$ I/D and ACE G2350A allele and/or genotype combinational calculations associated with hypertension and sports performance are summarized in Tables 4A \& 4B, respectively.

Table 4A: Summary of the association between hypertension and genotype combinations of the both ACE I/D and ACE G2350A polymorphisms.

\begin{tabular}{|c|c|c|c|c|c|}
\hline ACE genes & Allele \& Genotype & OR & $95 \% \mathrm{CI}$ & $\chi^{2}$ & $\mathbf{p}$ \\
\hline \multirow[t]{5}{*}{$A C E \mathrm{I} / \mathrm{D}$} & I vs. D & 0.902 & $1.01-2.76$ & 0.051 & 0.821 \\
\hline & D vs. I & 1.108 & $1.01-2.76$ & 0.051 & 0.821 \\
\hline & II vs. ID+DD & 3.545 & $1.03-14.41$ & 1.130 & 0.287 \\
\hline & ID vs. II+DD & 0.394 & $1.01-3.90$ & 2.349 & 0.125 \\
\hline & DD vs. II+ID & 1.650 & $1.01-3.56$ & 0.778 & 0.377 \\
\hline \multirow[t]{3}{*}{$A C E \mathrm{G} 2350 \mathrm{~A}$} & G vs. A & 0.988 & $1.01-2.41$ & 0.00094 & 0.975 \\
\hline & A vs. G & 1.011 & $1.01-2.41$ & 0.00077 & 0.977 \\
\hline & GG vs. GA+AA & 0.520 & $1.04-16.75$ & 0.270 & 0.603 \\
\hline
\end{tabular}




\begin{tabular}{|l|l|c|c|c|c|}
\hline & GA vs. GG+AA & 1.5 & $1.02-4.97$ & 0.321 & 0.571 \\
\hline & AA vs. GG+GA & 0.784 & $1.02-6.28$ & 0.088 & 0.766 \\
\hline
\end{tabular}

Table 4B: Summary of the association between sport performance and genotype combinations of the both ACE I/D and ACE G2350A polymorphisms.

\begin{tabular}{|c|c|c|c|c|c|}
\hline ACE genes & Allele \& Genotype & OR & $95 \% \mathrm{CI}$ & $\chi^{2}$ & $\mathbf{p}$ \\
\hline \multirow[t]{5}{*}{$A C E \mathrm{I} / \mathrm{D}$} & I vs. D & 1.234 & $1.01-2.88$ & 0.197 & 0.657 \\
\hline & D vs. I & 0.810 & $1.01-2.88$ & 0.198 & 0.656 \\
\hline & II vs. ID+DD & 3.545 & $1.03-14.41$ & 1.130 & 0.287 \\
\hline & ID vs. II+DD & 0.631 & $1.01-4.02$ & 0.549 & 0.458 \\
\hline & DD vs. II+ID & 1.045 & $1.01-3.64$ & 0.0058 & 0.939 \\
\hline \multirow[t]{5}{*}{ ACE G2350A } & G vs. A & 1.338 & $1.01-2.44$ & 0.535 & 0.464 \\
\hline & A vs. $G$ & 0.747 & $1.01-2.44$ & 0.537 & 0.463 \\
\hline & GG vs. GA+AA & N.A.* & N.A.* & N.A.* & N.A.* \\
\hline & GA vs. GG+AA & 1.5 & $1.02-4.97$ & 0.321 & 0.571 \\
\hline & AA vs. GG+GA & 0.477 & $1.02-5.61$ & 0.923 & 0.336 \\
\hline
\end{tabular}

OR: odds ratio, 95\%CI: confidence interval, $\chi^{2}$ : Pearson's chi-squared test, p: probability value

*: There is not any person who carry GG genotype in elite athletes group.

Haplotype Association of ACE I/D and ACE G2350A

\section{Polymorphisms in $\mathrm{H}, \mathrm{E}$ and C Groups}

The haplotype frequencies of the ACE rs1799752 (I/D) and $A C E$ rs4343 (G2350A) polymorphisms in the study groups are presented in Table 5. Haplotype analysis showed that, using rs1799752-rs4343 haplotype I-G as reference, the frequency of haplotype D-A, I-A, D-G had no significant differences in between hypertension patients, elite athletes and healthy controls $34.72 \%$ vs. $36.84 \%$ for haplotype D-A, $\chi^{2}$ (p-value) $=0.135$ (0.713), OR
$(95 \% \mathrm{CI})=0.824(1.016-3.243)$ in $\mathrm{H}$ vs. C; $40 \%$ vs. $36.84 \%$ for haplotype D-A in E vs. C, $\chi^{2}$ (p-value) $=0.147$ (0.701), OR $(95 \% \mathrm{CI})=$ 1.238 (1.017-3.471); $16.67 \%$ vs. $14.04 \%$ for haplotype I-A in H vs. $\mathrm{C}, \chi^{2}(\mathrm{p}$-value $)=0.0035(0.952)$, OR $(95 \% \mathrm{CI})=1.038(1.019-4.10)$; $15.38 \%$ vs. $14.04 \%$ for haplotype I-A in E vs. C, $\chi^{2}$ (p-value) $=0.111$ (0.739), OR (95\%CI) = $1.25(1.021-4.476) ; 30.56 \%$ vs. $33.33 \%$ for haplotype D-G in H vs. C, $\chi^{2}$ (p-value) = $0.172(0.678), 0 R(95 \% \mathrm{CI})=$ 0.801 (1.016-3.316); 30.77\% vs. 33.33\% for haplotype D-G in E vs. C, $\chi^{2}(\mathrm{p}$-value $)=0.0079(0.929)$, OR $(95 \% \mathrm{CI})=1.052(1.018-3.587)$; respectively, Table 5).

Table 5: Haplotype frequencies of ACE rs1799752 (I/D) and ACE rs4343 (G2350A) polymorphisms in patients with hypertension, elite athletes and healthy controls.

\begin{tabular}{|c|c|c|c|c|c|c|c|c|c|c|}
\hline \multirow[b]{2}{*}{$\begin{array}{c}\text { ACE } \\
\text { polymorphism }\end{array}$} & \multirow[b]{2}{*}{$\begin{array}{c}H \\
(n=27)\end{array}$} & \multirow[b]{2}{*}{$\begin{array}{c}\text { H } \\
\text { Genotype } \\
\text { frequen- } \\
\text { cies }\end{array}$} & \multirow[b]{2}{*}{$\begin{array}{c}E \\
(n=27)\end{array}$} & \multirow[b]{2}{*}{$\begin{array}{l}\text { E Geno- } \\
\text { type fre- } \\
\text { quencies }\end{array}$} & \multirow[b]{2}{*}{$\begin{array}{c}C \\
(n=25)\end{array}$} & \multirow[b]{2}{*}{$\begin{array}{c}\text { C } \\
\text { Genotype } \\
\text { frequen- } \\
\text { cies }\end{array}$} & \multicolumn{2}{|c|}{$\begin{array}{c}\text { Hypertension Patients } \\
\text { vs. Controls }\end{array}$} & \multicolumn{2}{|c|}{ Elite athletes vs. Controls } \\
\hline & & & & & & & $\begin{array}{c}\text { Chi square } \\
\text { test for } \\
\text { association } \\
\chi^{2}(p)\end{array}$ & $\begin{array}{c}\text { OR } \\
(95 \% \mathrm{CI})\end{array}$ & $\begin{array}{c}\text { Chi square } \\
\text { test for } \\
\text { association } \\
\chi^{2}(p)\end{array}$ & OR $(95 \% \mathrm{CI})$ \\
\hline \multicolumn{11}{|c|}{ Haplotype (rs1799752-rs4343, ACE ID / G2350A) } \\
\hline $\begin{array}{c}\mathrm{I}-\mathrm{G} \\
(\mathrm{II}+\mathrm{GG}, \mathrm{ID}+\mathrm{GA}, \\
\mathrm{II}+\mathrm{GA}, \mathrm{ID}+\mathrm{GG})\end{array}$ & 13 & $18.05 \%$ & 9 & $13.85 \%$ & 9 & $15.79 \%$ & \multicolumn{2}{|c|}{ Reference } & \multicolumn{2}{|c|}{ Reference } \\
\hline $\begin{array}{c}\mathrm{D}-\mathrm{A} \\
(\mathrm{DD}+\mathrm{AA}, \mathrm{ID}+\mathrm{GA}, \\
\mathrm{DD}+\mathrm{GA}, \mathrm{ID}+\mathrm{AA})\end{array}$ & 25 & $34.72 \%$ & 26 & $40 \%$ & 21 & $36.84 \%$ & $\begin{array}{c}0.135 \\
(0.713)\end{array}$ & $\begin{array}{r}0.824 \\
(1.016- \\
3.243) \\
\end{array}$ & $\begin{array}{c}0.147 \\
(0.701)\end{array}$ & $\begin{array}{r}1.238 \\
(1.017- \\
3.471) \\
\end{array}$ \\
\hline $\begin{array}{c}\mathrm{I}-\mathrm{A} \\
(\mathrm{II}+\mathrm{AA}, \mathrm{ID}+\mathrm{GA}, \\
\mathrm{II}+\mathrm{GA}, \mathrm{ID}+\mathrm{AA})\end{array}$ & 12 & $16.67 \%$ & 10 & $15.38 \%$ & 8 & $14.04 \%$ & $\begin{array}{l}0.0035 \\
(0.952)\end{array}$ & $\begin{array}{c}1.038 \\
(1.019- \\
4.10) \\
\end{array}$ & $\begin{array}{c}0.111 \\
(0.739)\end{array}$ & $\begin{array}{c}1.25 \\
(1.021- \\
4.476) \\
\end{array}$ \\
\hline $\begin{array}{c}D-G \\
(D D+G G, I D+G A, \\
D D+G A, I D+G G)\end{array}$ & 22 & $30.56 \%$ & 20 & $30.77 \%$ & 19 & $33.33 \%$ & $\begin{array}{c}0.172 \\
(0.678)\end{array}$ & $\begin{array}{c}0.801 \\
(1.016- \\
3.316)\end{array}$ & $\begin{array}{l}0.0079 \\
(0.929)\end{array}$ & $\begin{array}{l}1.052 \\
(1.018- \\
3.587)\end{array}$ \\
\hline
\end{tabular}


Genetic association of rs1799752 (ACE I/D) and rs4343 (ACE G2350A) polymorphisms in Hypertension patients, Elite athletes and healthy controls

Chi-square $\left(\chi^{2}\right)$ Test for Independence was used to determine whether could be a significant association between patients with hypertension, elite athletes and healthy controls. $\chi^{2}$ independence test was conducted for ACE I/D and ACE G2350A polymorphisms, respectively. $\chi^{2}$ independence and degrees of freedom (df) values were determined by direct counting method on all groups. P values for $\mathrm{c}^{2}$ test for independence were calculated by using R i386 (X64) 3.4.1. platform (Table 6). The $\chi^{2}$ test for independence analysis of rs1799752 (ACE I/D) and rs4343 (ACE G2350A) polymorphisms in between patients with hypertension and elite athletes had no significant differences $\left(\chi^{2}\right.$ independence $=0.78, \mathrm{df}=2, \mathrm{p}$-value $=$ 0.698 for $A C E \mathrm{I} / \mathrm{D} ; \chi^{2}$ independence $=2.4, \mathrm{df}=2, \mathrm{p}$-value $=0.301$ for ACE G2350A). However, there was no significant differences supporting association of rs1799752 and rs4343 polymorphisms between $\mathrm{H}$ vs. C and E vs. C, respectively ( $\mathrm{H}$ vs. C, $\chi^{2}$ independence $=3.059, \mathrm{df}=2, \mathrm{p}$-value $=0.216 ; \mathrm{E}$ vs. $\mathrm{C}, \chi^{2}$ independence $=1.556, \mathrm{df}$ $=2$, $\mathrm{p}$-value $=0.459$ for $A C E \mathrm{I} / \mathrm{D}$; H vs. C, $\chi^{2}$ independence $=0.4, \mathrm{df}=$ $2, \mathrm{p}$-value $=0.818 ; \mathrm{E}$ vs. $\mathrm{C}, \chi^{2}$ independence $=1.915, \mathrm{df}=2, \mathrm{p}$-value $=$ 0.383 for ACE G2350A, Table 6).

Table 6A: Chi-square $\mathrm{x}^{2}$ test for independence results for $A C E$ rs1799752 (I/D) and ACE rs4343 (G2350A) polymorphisms.

\begin{tabular}{|c|c|c|c|}
\hline & $\begin{array}{c}\chi^{2} \\
\text { independence }\end{array}$ & $\begin{array}{c}\text { df } \\
\text { (degrees } \\
\text { of } \\
\text { freedom) }\end{array}$ & P-value \\
\hline $\begin{array}{c}\text { Hypertension patients, } \\
\text { Elite athletes, Controls (n } \\
=79 \text { ) }\end{array}$ & 4.595 & 4 & 0.331 \\
\hline $\begin{array}{c}\text { Hypertension patients vs. } \\
\text { Controls (n = 52) }\end{array}$ & 3.059 & 2 & 0.216 \\
\hline $\begin{array}{c}\text { Elite athletes vs. Controls } \\
\text { (n=52) }\end{array}$ & 1.556 & 2 & 0.459 \\
\hline $\begin{array}{c}\text { Hypertension patients vs. } \\
\text { Elite athletes (n = 54) }\end{array}$ & 0.718 & 2 & 0.698 \\
\hline
\end{tabular}

Note: rs1799752 (ACE I/D) « $\chi^{2}$ independence test» results.

Table 6B: Chi-square $\chi^{2}$ test for independence results for $A C E$ rs1799752 (I/D) and ACE rs4343 (G2350A) polymorphisms.

\begin{tabular}{|c|c|c|c|}
\hline & $\chi^{2}$ independence & $\begin{array}{c}\text { df (degrees } \\
\text { of freedom) }\end{array}$ & P-value \\
\hline $\begin{array}{c}\text { Hypertension patients, } \\
\text { Elite athletes, Controls } \\
(\mathrm{n}=79)\end{array}$ & 2.937 & 4 & 0.568 \\
\hline $\begin{array}{c}\text { Hypertension patients } \\
\text { vs. Controls (n= 52) }\end{array}$ & 0.4 & 2 & 0.818 \\
\hline
\end{tabular}

\begin{tabular}{|c|c|c|c|}
\hline $\begin{array}{c}\text { Elite athletes vs. Controls } \\
(\mathrm{n}=52)\end{array}$ & 1.915 & 2 & 0.383 \\
\hline $\begin{array}{c}\text { Hypertension patients } \\
\text { vs. Elite athletes }(\mathrm{n}=54)\end{array}$ & 2.4 & 2 & 0.301 \\
\hline
\end{tabular}

Note: rs4343 (ACE G2350A) « $\chi^{2}$ independence test» results.

\section{Discussion}

Hypertension is an important risk factor for a variety of cardiovascular, renal and neurological diseases. Elevated blood pressure (BP) is a precursor to excessive morbidity and premature mortality. Although systemic hypertension is a risk factor for disease burden, the risk is uneven, heterogeneous and unpredictable. Also, genetic factors contribute to $30-50 \%$ of causation to essential hypertension [10]. Hypertension may be identified as a polygenic hereditary disease simultaneously influenced by a variety of environmental factors [11]. Therefore, it raises the possibility of genetics in the development of hypertension and/or related complications [12]. The renin-angiotensin-aldosterone system (RAAS) contributes to electrolyte homeostasis and regulation of blood pressure. Angiotensin-converting enzyme (ACE) is the key enzyme of the RAAS. An imbalance between forces of vasoconstriction over forces of vasodilation elevates vascular tone and leads to systemic elevation of BP. Based on this theory, ACE gene polymorphisms associated with high (or inappropriate) levels of $A C E$ can be considered as a genetic model in the development of hypertension and its complications [13].

In this study, it was investigated the role of $A C E$ I/D and $A C E$ G2350A polymorphisms on hypertension and sport performance in the Turkish population. The subjects with already known health problem other than essential hypertension were excluded from the study for avoiding any potential confounding effects. Insertion/deletion (I/D) and G2350A polymorphisms of the $A C E$ gene have been studied about cardiovascular diseases, especially hypertension $[14,15]$. ACE genotype studies have revealed several different results due to variable frequency of the related $A C E$ alleles (I/D, G/A) among populations. In past, ACE I/D polymorphism has been extensively studied in association with hypertension [16]. A large meta-analysis by Staessen et al. [17] revealed that homozygous D allele causes 10\% increased risk for hypertension when compared to that of homozygous I allele in women and Asians. For the Hispanic population, DD genotype was found independent risk factor of hypertension [18]. Similar results were observed in the population of China, the male population of Bangladesh, Japan and Argentina [19-22]. These results were not replicated in other similar investigations. For example, a meta-analysis restricted to Caucasians showed no association of ACE I/D polymorphism with hypertension [23]. Similar reports were submitted by Miyama et al. [24] and Glavnik and Petrovic [25]. For the Pakistani population, two studies were noted in this regard. Ismail et al. [26] reported that ACE II genotype is associated with essential hypertension in young Pakistani population, but this association was not observed 
by Alvi and Hasnain [15].

The polymorphism in exon $17, A C E$ G2350A has also reported being associated with plasma ACE concentration. Zhu et al. [27] have observed its association with hypertension. In the population of Emirati, similar results were noted by Saeed Mahmood et al. [9] using a sample population of 254 individuals. Nawaz and Hasnain also observed an association between ACE G2350A polymorphism and hypertension in a different research project.

Also, Nawaz and Hasnain investigated the role of ACE I/D and ACE G2350A polymorphisms in increasing the blood pressure of subjects exposed to different sound levels. Their findings suggest that noise has no prominent role in changing the effects of $A C E$ I/D polymorphism for increasing the blood pressure [28]. It was observed that different allele carriers of the ACE I/D and $A C E$ G2350A gene polymorphisms have different chances of hypertension on exposure to noise [12].

In this study, the distribution of the II, ID and DD genotypes of the H-E-C subjects was $0.03,0.44,0.51(\mathrm{H}) ; 0.03,0.33,0.62$ (E); $0.12,0.24,0.64$ (C), respectively. The number of people who carry the ACE "D" allele (ID+DD genotypes) was higher among patients with hypertension (0.95) and elite athletes (0.95) than $A C E$ II genotype carriers. Otherwise, we couldn't find a statistically significant association for ACE I/D polymorphism among our study groups ( $\mathrm{p}>0.05)$. For the association between ACE G2350A polymorphism and hypertension / sport performance, the distribution of the GG, GA and AA genotypes of the study subjects was assessed as $0.07,0.77,0.14(\mathrm{H}) ; 0,0.77,0.22(\mathrm{E})$ and $0.04,0.84$, 0.12 (C), respectively. The number of $A C E$ " $\mathrm{A}$ " allele carriers (GA+AA genotypes) was higher (0.92-H, 1-E) than the subjects who carry the ACE GG genotype. Also, it was not found any significant association between $\mathrm{H}-\mathrm{C}$ and E-C groups ( $\mathrm{p}>0.05$ ). Fajar et al. [29] reviewed a meta-analysis to evaluate the correlation between $A C E$ I/D and $A C E$ G2350A polymorphisms for left ventricular hypertrophy. Previous studies had shown that $\mathrm{D}$ allele of $A C E \mathrm{I} / \mathrm{D}$ was associated with an elevated level of $A C E$ in the circulation [30-33] and increased $A C E$ mRNA expressions [34] and activity [35]. Also, it was demonstrated that $\mathrm{G}$ allele of $A C E$ G2350A was associated with an elevated $A C E$ level in the circulation $[9,12]$. According to the Fajar's pooled data, D allele of ACE I/D was associated with 1.26-fold and A allele of ACE G2350A was correlated with 1.67-fold increasing the risk of left ventricular hypertrophy. Compared to "D" allele of ACE I/D, "A" allele of $A C E$ G2350A had a greater risk for left ventricular hypertrophy. In our study, we showed "D" allele of ACE I/D was associated with 0.902-fold for $(\mathrm{H})$ and 1.234-fold for (E) subjects, respectively. Also, it was found for "A" allele of ACE G2350A that 0.988-fold (H) and 1.338-fold (E) increasing the risk of hypertension and sports performance, respectively. The present study was conducted in small sample sizes of patients and controls, genotyped and analyzed only two polymorphisms (rs1799752 and rs4343) in the
$A C E$ gene. Further studies in large patient and control populations are needed to elucidate the possible role of $A C E$ polymorphisms in hypertension risk.

\section{Conclusion}

In conclusion, this study was examined the possible association and haplotypes formed by rs1799752 (ACE I/D) and rs4343 (ACE G2350A) polymorphisms on essential hypertension patients, elite athletes and healthy controls in Turkish population. Carriers of the DD and ID genotypes of the ACE I/D gene and GA genotype of the ACE G2350A gene had a higher frequency in all three study groups. These findings suggest it could be a possible role of these two polymorphisms (D allele for ACE I/D and A allele for $A C E$ G2350A polymorphism) in Turkish population, but there was not found statistically significant differences in between hypertension patients and elite athletes compared by controls.

\section{Ethics Approval and Consent to Participate}

The study was approved by the Ethics Committee of Istanbul University (Istanbul, Turkey; Project no. 2011/2115-897).

\section{Patient Consent for Publication}

All the participants provided written informed consent.

\section{References}

1. Ann JH, Ko SK (2002) The change of the cardiac structure and function on the static and dynamic training. Exerc Sci 11(1): 189-198.

2. Jang Y, Kim SM (2012) Influences of the G2350A polymorphism in the $A C E$ Gene on cardiac structure and function of ball game players. J Negat Results Biomed 11(6): 1-7.

3. Cauci S, Di Santolo M, Ryckman KK, Williams SM, Banfi G (2010) Variable number of tandem repeat polymorphisms of the interleukin-1 receptor antagonist gene IL-1RN: a novel association with the athlete status. BMC Med Genomics 11(29): 1-11.

4. Fatini CF, Guazzelli R, Manetti P, Battaglini B, Gensini F, et al. (2000) RAS gene influence exercise-induced left ventricular hypertrophy: an elite athletes study. Med Sci Sports Exerc 32: 1868-1872.

5. Sayed Tabatabaei FA, Oostra BA, Isaacs A, Van Duijn CM, Witteman JC (2006) ACE Polymorphisms. Circ Res 98(9): 1123-1133.

6. Pan M, Zhu JH, Liu ZH, Jiang WP, Cui ZC, et al. (2007) Angiotensinconverting enzyme gene $2350 \mathrm{G} / \mathrm{A}$ polymorphism is associated with left ventricular hypertrophy but not essential hypertension. Hypertens Res 30(1): 31-37.

7. Niu W, Qi Y, Hou S, Zhai X, Zhou W, et al. (2009) Haplotype-based association of the renin-angiotensin-aldosterone system genes polymorphisms with essential hypertension among Han Chinese: the Fangshan study. J Hypertens 27(7): 1384-1391.

8. Niu W, Qi Y, Gao P, Zhu D (2011) Review: Association between angiotensin converting enzyme G2350A polymorphism and hypertension risk: a meta-analysis. J Renin Angiotensin Aldosterone Syst 12(1): 8-14.

9. Saeed Mahmood M, Saboohi K, Osman Ali S, Bokhari AM, Frossard PM (2003) Association of the angiotensin-converting enzyme (ACE) gene G2350A dimorphism with essential hypertension. J Hum Hypertens 17(10): 719-723.

10. Mancia G, De Backer G, Dominiczak A, Cifkova R, Fagard R, et al. (2007) Guidelines for the management of arterial hypertension: The task force 
for the management of arterial hypertension of the European Society of Hypertension (ESH) and of the European Society of Cardiology (ESC). Eur Heart J 28(12): 1462-1536.

11. Siddhart KM, Kapur S, Venkata S, Ram C (2012) Angiotensin converting enzyme gene polymorphism and hypertension: no $A C E$ yet in the pack of cards. J Assoc Physicians India 60(6): 9-10.

12. Zawilla N, Shaker D, Abdelaal A, Aref W (2014) Angiotensin-converting enzyme gene polymorphisms and hypertension in occupational noise exposure in Egypt. Int J Occup Environ Health 20(3): 194-206.

13. Badaruddoza AJS, Bhanwer R, Sawhney NK, Randhawa K, Barna B (2009) A study of angiotensin converting enzyme $(A C E)$ gene polymorphism in essential hypertension among a business community in Punjab. Int J Hum Genet 9(3-4): 231-234.

14. Kim K (2009) Association of angiotensin-converting enzyme insertion/ deletion polymorphism with obesity, cardiovascular risk factors and exercise-mediated changes in Korean women. Eur J Appl Physiol 105(6): 879-887.

15. Alvi FM, Hasnain S (2009) ACE I/D and G2350A polymorphisms in Pakistani hypertensive population of Punjab. Clin Exp Hypertens 31(5): 471-480.

16. Nawaz SK, Hasnain S (2009) Pleiotropic effects of ACE polymorphism Biochemia Medica 19(1): 36-49.

17. Staessen JA, Wang JG, Ginocchio G, Petrov V, Saavedra AP, et al. (1997) The deletion/insertion polymorphism of the angiotensin converting enzyme gene and cardiovascularrenal risk. J Hypertens 15(2): 15791592.

18. Bautista LE, Vargas CI, Oróstegui M, Gamarra G (2008) Population based case-control study of renin-angiotensin system genes polymorphisms and hypertension among Hispanics. Hypertens Res 31(3): 401-408.

19. Zhang YL, Zhou SX, Lei J, Zhang JM (2007) Association of angiotensin I-converting enzyme gene polymorphism with $A C E$ and PAI-1 levels in Guangdong Chinese Han patients with essential hypertension. Nan Fang Yi Ke Da Xue Xue Bao 27(11): 1681-1684.

20. Morshed M, Khan H, Akhteruzzaman S (2002) Association between angiotensin I-converting enzyme gene polymorphism and hypertension in selected individuals of the Bangladeshi population. J Biochem Mol Biol 35(3): 251-254.

21. Higaki J, Baba S, Katsuya T, Sato N, Ishikawa K, et al. (2000) Deletion allele of angiotensin-converting enzyme gene increases risk of essential hypertension in Japanese men: the suita study. Circulation 101(17): 2060-2065.

22. Jiménez PM, Conde C, Casanegra A, Romero C, Tabares AH, et al. (2007) Association of $A C E$ genotype and predominantly diastolic hypertension: a preliminary study. J Renin Angiotensin Aldosterone Syst 8(1): 42-44.

23. Agerholm Larsen B, Nordestgaard BG, Tybjaerg Hansen A (2000) ACE gene polymorphism in cardiovascular disease: meta-analyses of small and large studies in whites. Arterioscler Thromb Vasc Biol 20(2): 484492.

\section{ISSN: 2574-1241}

DOI: 10.26717/BJSTR.2020.31.005032

Tuba Gunel. Biomed J Sci \& Tech Res

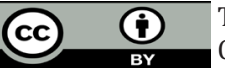

This work is licensed under Creative

Commons Attribution 4.0 License

Submission Link: https://biomedres.us/submit-manuscript.php
24. Miyama N, Hasegawa Y, Suzuki M, Hida W, Kazama I, et al. (2007) Investigation of major genetic polymorphisms in the renin-angiotensinaldosterone system in subjects with young-onset hypertension selected by a targeted-screening system at university. Clin Exp Hypertens 29(1): 61-67.

25. Glavnik N, Petrovic D (2007) M235T polymorphism of the angiotensinogen gene and insertion/deletion polymorphism of the angiotensin-1 converting enzyme gene in essential arterial hypertension in Caucasians. Folia Biol (Praha) 53(2): 69-70.

26. Ismail M, Akhtar N, Nasir M, Firasat S, Ayub Q et al. (2004) Association between the angiotensin-converting enzyme gene insertion/deletion polymorphism and essential hypertension in young Pakistani patients. I Biochem Mol Biol 37(5): 552-555.

27. Zhu X, Bouzekri N, Southam L, Cooper RS, Adeyemo A, et al. (2001) Linkage and association analysis of angiotensin I-converting enzyme $(A C E)$ - gene polymorphisms with $A C E$ concentration and blood pressure. Am J Hum Genet 68(5): 1139-1148.

28. Nawaz SK, Hasnain S (2011) Association of ACE ID and ACE G2350A polymorphism with increased blood pressure in persons exposed to different sound levels in Pakistan. Int Arch Occup Environ Health 84(4): 355-360.

29. Fajar JK, Pikir BS, Sidarta EP, Saka PNB, Akbar RR, et al. (2019) The genes polymorphism of angiotensin-converting enzyme (ACE) I/D and $A C E$ G2350A in patients with left ventricular hypertrophy: a meta-analysis. Indian Heart Journal (IHJ) 71(3): 199-206

30. Foy CA, Mc Cormack LJ, Knowler WC, Barrett JH, Catto A, et al. (1996) The angiotensin-I converting enzyme (ACE) gene I/D polymorphism and $A C E$ levels in Pima Indians. J Med Genet 33(4): 336-337.

31. Montgomery HE, Clarkson P, Dollery CM, Prasad K, Losi MA, et al. (1997) Association of angiotensin-converting enzyme gene I/D polymorphism with change in left ventricular mass in response to physical training. Circulation 96(3): 741-747.

32. Das S, Roy S, Sharma V, Kaul S, Jyothy A, et al. (2015) Association of $A C E$ gene I/D polymorphism and $A C E$ levels with hemorrhagic stroke: comparison with ischemic stroke. Neurol Sci 36(1): 137-142.

33. Kostadinova ES, Miteva LD, Stanilova SA (2017) ACE serum level and I/D gene polymorphism in children with obstructive uropathies and other congenital anomalies of the kidney and urinary tract. Nephrology (Carlton) 22(8): 609-616.

34. Mizuiri S, Hemmi H, Kumanomidou H, Iwamoto M, Miyagi M, et al. (2001) Angiotensin-converting enzyme ( $A C E)$ I/D genotype and renal $A C E$ gene expression. Kidney Int 60(3): 1124-1130.

35. Ajala AR, Almeida SS, Rangel M, Palomino Z, Strufaldi MW, et al. (2012) Association of $A C E$ gene insertion/deletion polymorphism with birth weight, blood pressure levels, and $A C E$ activity in healthy children. Am J Hypertens 25(7): 827-832.

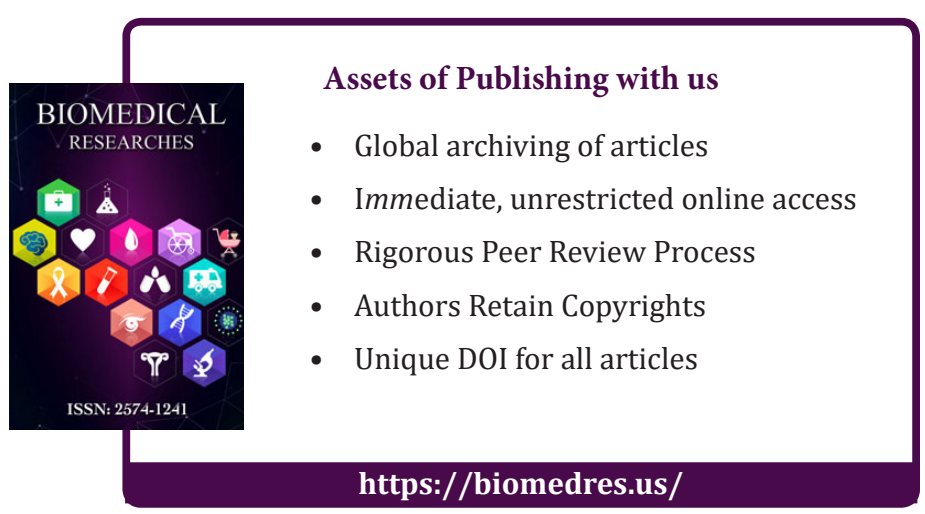

Copyright@ Tuba Gunel | Biomed J Sci \& Tech Res | BJSTR. MS.ID.005032. 